\title{
Harnessing Cellular-Derived Forces in Self-Assembled Microtissues to Control the Synthesis and Alignment of ECM
}

\author{
Jacquelyn Y.Schell ${ }^{1,2}$, Benjamin T. Wilks ${ }^{1,2}$, Mohak Patel ${ }^{3}$, Christian Franck ${ }^{3}$, Vijaya
} Chalivendra $^{4}$, Xuan Cao ${ }^{5}$, Vivek B. Shenoy ${ }^{5}$, and Jeffrey R. Morgan ${ }^{1,2}$.

\section{Author Affiliation:}

${ }^{1}$ Department of Molecular Pharmacology, Physiology and Biotechnology Brown University, Providence, RI, USA

${ }^{2}$ Center for Biomedical Engineering, Brown University, Providence, RI, USA

${ }^{3}$ School of Engineering, Brown University, Providence, RI, USA

${ }^{4}$ Department of Mechanical Engineering, University of Massachusetts Dartmouth, North Dartmouth, Massachusetts

${ }^{5}$ Department of Bioengineering University of Pennsylvania Philadelphia, PA 19104

\section{Corresponding Author:}

Jeffrey R. Morgan

Brown University, G-B 393, Biomed Center

171 Meeting St., Providence, RI 02912

Tel: 401-863-9879. Fax: 401- 863-1753

E-mail: Jeffrey_Morgan@Brown.edu 


\begin{abstract}
The alignment and blend of extracellular matrix (ECM) proteins give a tissue its specific mechanical properties as well as its physiological function. Various tissue engineering methods have taken purified ECM proteins and aligned them into gels, sponges and threads. Although, each of these methods has created aligned ECM, they have had many limitations including loss of hierarchal collagen structure and poor mechanical performance. Here, we have developed a new method to control ECM synthesis using self-assembled cells. Cells were seeded into custom designed, scaffold-free, micro-molds with fixed obstacles that harnessed and directed cellmediated stresses. Cells within the microtissue reacted to self-generated tension by aligning, elongating, and synthesizing an ECM whose organization was dictated by the strain field that was set by our micro-mold design. We have shown that through cell selection, we can create tissues with aligned collagen II or aligned elastin. We have also demonstrated that these selfassembled microtissues have mechanical properties in the range of natural tissues and that mold design can be used to further tailor these mechanical properties.
\end{abstract}

\title{
Keywords
}

Self-assembly; spheroid; cell-derived ECM; mechanical properties; strong tissues; aligned ECM 


\section{Introduction}

The extracellular matrix (ECM) is a complex biocomposite that serves diverse functions including the provision of biological cues and mechanical support for cells. This complex interplay between the structure and composition of the ECM is crucial to the utility of a tissue as a whole. For decades, investigators have been trying to recapitulate the complexity of the ECM with ambitions of creating natural and strong tissue equivalents/biomaterials with precise spatial organization and alignment. Collagen, silk, fibrin, and elastin are some of the naturally derived polymers that have been fabricated, with various levels of success, into aligned biomaterials/tissues (1-5). Much research has focused on collagen because of its abundance and because early successes demonstrated its biocompatibility and ability to restore cells to a more natural phenotype (6). Although purified collagen has been successfully fabricated and aligned into a variety of materials including gels, porous sponges, and threads, and has been populated by a variety of cell types, major barriers arise when using purified collagen (7). Specifically, biomaterials made from purified collagen are far weaker than natural tissues, they lose their complex hierarchal structure and natural cross-links, and they do not have the biocomplexity (composition, blend) of the ECM that cells synthesize (8-10).

On the other end of the spectrum, researchers have investigated decellularizing natural tissues and organs. These materials have preserved much of the precise structural architecture of the ECM and can serve as complex scaffolds for cell attachment or cell growth $(11,12)$.

However, there are serious limitations with decellularized organs including availability, lot-to-lot variability, and transmission of diseases. Tissue grafts obtained from cadavers have inherent variability due to the fact that each graft is from a unique donor (genetics, age, size, shape, medical history, etc.) as well as a unique anatomical site (or maximum of two) of that donor. 
And, although these materials can be crudely processed (cutting, shaping) there is no possibility to truly engineer their underlying mechanical or biological composition. Missing is a natural, strong, material that can be tailored in terms of mechanical properties, composition, and architecture.

An increasing amount of evidence has demonstrated that self-assembled microtissues more closely match the complexity of in vivo tissues including the architecture, differentiated functions, metabolic activity as well as the synthesis of ECM (13-17). We have previously demonstrated that through micro-mold design, we can form complex shaped microtissues such as toroids, rods, honeycombs, and loop ended dogbones (18-20). We have also demonstrated that self-assembling cells generate measurable contractile forces and that micro-molds can harness these cell-mediated stresses during self-assembly and direct the strain that develops in the resulting tissue $(21,22)$. Specifically, we have shown that when cells are seeded in a loop-ended dogbone recess, the cells will align and stretch uniaxially. Our hypothesis is that micro-mold design dictates cell alignment which in turn controls the alignment of the ECM that these cells synthesize and this ECM alignment dictates the mechanical properties of the tissue. The objectives of this study was to form self-assembled microtissues in five different mold designs, characterize the cell and ECM alignment in these five designs and test mechanical properties.

Here, we have shown that by simply altering mold design (trough size/shape and peg constraint placement) we can recapitulate a number of tension environments to create aligned tissues. We designed different micro-molds to create tissues with uniaxial tension (loop-ended dogbones tissues), circumferential tension (toroid tissues), biaxial tension (cross tissues), equibiaxial tension (trampoline tissue), and multiaxial tension (triangle tissue) as these alignments represent a number of alignments that are found in natural tissues. When cells were 
seeded into these non-adhesive, scaffold-free molds, they formed cell-cell adhesions, exerted contractile forces, aligned, and over time, formed aligned ECM. The alignment of the cells and their ECM varied according to the design of the micro-mold and the kind of tension that it was designed to induce in the tissue. The resulting tissues were strong and exhibited mechanical properties in the range of natural tissues. Furthermore, we demonstrated that through cell selection, we can elicit the synthesis of aligned collagen II and elastin, and through mold design, we can further tailor mechanical properties.

\section{Materials and Methods}

\section{Micro-Mold Design and Gel Casting}

Micro-molds were designed using the computer aided design (CAD) software SolidWorks (SolidWorks Corporation, Concord, MA). Dogbone molds have been previously described (22). Briefly, dogbone recesses consisted of two tori with inner diameters of $800 \mu \mathrm{m}$ and outer diameters of $2.4 \mathrm{~mm}$, set $5 \mathrm{~mm}$ apart and connected by a trough (connecting rod) of width $400 \mu \mathrm{m}$ or $600 \mu \mathrm{m}$. Toroid molds have also been previously described (19). Each toroid feature was a cylindrical well, $1.4 \mathrm{~mm}$ in diameter and contained a circular trough and a cylindrical peg that extended upward from the center of the circular trough. The circular trough was $400 \mu \mathrm{m}$ wide and the cylindrical peg was $600 \mu \mathrm{m}$ in diameter. The small trampoline mold was a hexagon shaped recess with six toroid like features at the vertices of the hexagon. The total surface area of the bottom of the small trampoline recess was $7.91 \mathrm{~mm}^{2}$. The cross molds were designed as a single cylindrical well with four pegs spaced $500 \mu \mathrm{m}$ from the cylinder's edge aligned in a cross formation at $0^{\circ}, 90^{\circ}, 180^{\circ}$ and $270^{\circ}$. The cross feature had a bottom surface area of $8.2 \mathrm{~mm}^{2}$. The triangle mold was an equilateral triangle (bottom surface area of $4.1 \mathrm{~mm}^{2}$ ) with rounded vertices that contained pegs $400 \mu \mathrm{m}$ from the edge of the vertices. The large 
trampoline molds were designed as a hexagonal shaped tissue with three outer orbitals of pegs spaced $600 \mu \mathrm{m}$ apart. The central portion of the large trampoline had a surface area of $22 \mathrm{~mm}^{2}$.

CAD files were used to produce thermowax molds with a rapid prototyping machine (3D Systems Corporation, Valencia, CA). Wax molds were replicated using OOMOO® 25 Silicone Rubber (Smooth-On, East Texas, PA). Silicone molds were submerged overnight in 100\% ethanol, rinsed in distilled water, and autoclaved. To create agarose hydrogel micro-molds, $2 \%$ (weight/volume) sterile molten agarose was added to each silicone mold, allowed to gel, removed from the silicone molds, and then placed in a sterile 6-well tissue culture dish. The gels were rinsed with fresh culture medium and then equilibrated in culture media at $37^{\circ} \mathrm{C}$.

\section{Cell Culture and Micro-mold Seeding}

Human chondrocytes (passage 3-9) were purchased from PromoCell (Heidelberg, Germany) and were expanded in PromoCell Chondrocyte Growth Medium supplemented with $1 \%$ pen/strep at $37^{\circ} \mathrm{C}$ and $5 \% \mathrm{CO}_{2}$. Cells were removed from flasks using a standard trypsin process. Briefly, cells were exposed to $0.04 \%$ trypsin for 10 minutes, quenched with medium containing serum, spun down at 800 rpm for 6 minutes, resuspended, and counted. Dogbone molds contain 8 dogbone recesses per a mold, and each dogbone was seeded with 0.835 and 0.121 million cells for the $400 \mu \mathrm{m}$ and $600 \mu \mathrm{m}$ width dogbone molds, respectively. The small trampoline, triangle, toroid, and large trampoline, cross molds were seeded with $0.3,0.2,0.3$, and 2 million cells, respectively. After 30 minutes, $3 \mathrm{ml}$ of chondrogenic media (DMEM supplemented with 1\% pen/strep, $64 \mu \mathrm{g} / \mathrm{ml} \mathrm{L-ascorbic} \mathrm{acid} \mathrm{2-phosphate,} 200 \mu \mathrm{g} / \mathrm{ml}$ L-proline, 5 $\mathrm{mg} / \mathrm{ml}$ ITS Premix Universal Culture Supplement, $10 \mathrm{ng} / \mathrm{ml}$ human recombinant TGF- $\beta 1$, and 
$1.0 \mathrm{mM}$ dexamethasone) was added. Chondrocyte microtissues were fed every 2-3 days with 3 $\mathrm{ml}$ of chondrogenic media and were maintained at $37^{\circ} \mathrm{C}$ and $5 \% \mathrm{CO}_{2}$. All chondrocyte tissues (passage 3-9) matured in micromolds immunostained positive for collagen II, consistent with prior studies showing that chondrocyte phenotype is restored when cells cultured in $2 \mathrm{D}$ and are then cultured in $3 \mathrm{D}(23)$.

Normal human fibroblasts (NHFs) (passage 3-7) were purchased from PromoCell (Heidelberg, Germany) and were expanded in high glucose DMEM with $10 \%$ fetal bovine serum (FBS), $1 \%$ pen/strep at $37^{\circ} \mathrm{C}$ and $10 \% \mathrm{CO}_{2}$. Cells were removed from flasks using a standard trypsin process. Briefly, cells were exposed to $0.05 \%$ trypsin for 10 minutes, quenched with serum containing medium, spun down at $800 \mathrm{rpm}$ for 6 minutes, resuspended, and counted. NHFs were seeded in $400 \mu \mathrm{m}$ dogbone molds at a density of 0.871 million cells per dogbone and into small trampoline molds at a density of 0.3 million cells. After 30 minutes, $3 \mathrm{ml}$ of high glucose DMEM supplemented with 1\% pen/strep, $50 \mu \mathrm{g} / \mathrm{ml}$ L-ascorbic acid 2-phosphate, 30 $\mu \mathrm{g} / \mathrm{ml}$ L-proline, and $7.5 \mathrm{ng} / \mathrm{ml}$ human recombinant TGF- $\beta 1$ was added to each well and media was changed every $2-3$ days. NHF microtissues were maintained at $37^{\circ} \mathrm{C}$ and $10 \% \mathrm{CO}_{2}$.

$\underline{\text { Immunohistochemistry, Confocal Microscopy, and Nuclei Alignment Analysis }}$

To analyze nuclei alignment, chondrocyte microtissues were matured for 4 days and then fixed in 4\% paraformaldehyde. Microtissues were labeled with $0.3 \mathrm{mM}$ 4', 6-Diamidino-2Phenylindole, dihydrochloride (DAPI) and 6.6 $\mu \mathrm{M}$ Oregon Green ${ }^{\circledR} 488$ Phalloidin (Life Technologies, Grand Island, NY) and confocal images were captured with a Zeiss LSM 510 confocal microscope (Carl Zeiss MicroImaging, Thornwood, NY, USA). Using the analyze 
particle function in Image J Software (Rasband, W.S., ImageJ, NIH), an ellipse was fit to each nuclei, and the angle of the major axis of each ellipse was measured. Angle bins were set at $30^{\circ}$ intervals for the cross, trampoline, and triangle microtissues, and at $15^{\circ}$ intervals for the dogbone microtissues. For each field of view ( $\mathrm{n}=8,9,7$, and 6 for the dogbone, triangle, cross, and trampoline, respectively, with an average of 60 nuclei for each field of view) the number of nuclei in each bin was counted and the percentage of nuclei in each angle bin was averaged for all fields of view to create angle distribution histograms. For each shape, to determine if each angle bin had statistically more nuclei than each of the other bins, a student t-test (two-tail, unequal variance), was performed with a $95 \%$ confidence interval.

To analyze ECM synthesis, chondrocyte microtissues matured for 4 and 8 weeks were fixed with $4 \%$ paraformaldehyde and permeabilized for 30 minutes with $0.2 \%$ Triton $x-100$. Microtissues were then immunostained by incubation in a blocking solution containing PBS with $5 \%$ normal goat serum, $1 \%$ (weight/volume) bovine serum albumin, and $0.05 \%$ Triton $\mathrm{x}-100$ agitated for 3 hours on a shaker table $(120 \mathrm{rpm})$. Samples were then incubated with a rabbit anticollagen type II antibody (Abcam, Cambridge MA) $(20 \mu \mathrm{g} / \mathrm{ml})$, and a mouse anti-collagen type I antibody (Abcam, Cambridge MA) $(10 \mu \mathrm{g} / \mathrm{ml})$, agitated on a shaker table at room temperature for 2 hours, and then incubated for $2-3$ days at $4{ }^{\circ} \mathrm{C}$. The primary antibody was removed, samples were washed for 30 minutes (3x every 10 minutes on shaker table) in PBS, and then blocking solution was added. Samples were incubated in blocking solution for 3 hours at room temperature with shaking, and then incubated on a shaker table with a Cy2 goat anti-rabbit IgG secondary (Jackson ImmunoResearch Laboratories, West Grove, PA) (20 $\mu \mathrm{g} / \mathrm{ml})$ and a Cy3 goat anti-mouse IgG secondary (Jackson ImmunoResearch Laboratories, West Grove, PA) (20 $\mu \mathrm{g} / \mathrm{ml})$ for 2 hours, and then incubated for 2-3 days at $4{ }^{\circ} \mathrm{C}$. . Samples were taken out of the secondary 
solution, and washed for 1 hour (6x every 10 minutes on a shaker table). Chondrocyte samples were then labeled with $0.3 \mathrm{mM}$ DAPI.

NHF microtissues were matured for 4 weeks and followed the same fixation, permeabilization, immunostaining procedures described above, except they were immunostained with a rabbit anti-elastin antibody ( $20 \mu \mathrm{g} / \mathrm{ml})$ (Abcam, Cambridge MA) and a mouse anticollagen type I antibody (Abcam, Cambridge MA) $(10 \mu \mathrm{g} / \mathrm{ml})$. The secondary antibodies used

were a Cy2 goat anti-rabbit IgG secondary (Jackson ImmunoResearch Laboratories, West Grove, PA) $(20 \mu \mathrm{g} / \mathrm{ml})$ and a Cy3 goat anti-mouse IgG secondary (Jackson ImmunoResearch Laboratories, West Grove, PA) $(20 \mu \mathrm{g} / \mathrm{ml})$.

\section{Mechanical Testing}

To analyze the mechanical properties of self-assembled microtissues, dogbone microtissues with connecting rod widths of 400 and $600 \mu \mathrm{m}$ ( $\mathrm{n}=11$ and 5, respectively) were matured in chondrogenic media for 8 weeks. Prior to mechanical testing, each dogbone sample was imaged in its gel from the top and side using a Carl Zeiss Axio Observer Z1 microscope equipped with an AxioCam MRm camera (Carl Zeiss MicroImaging, Thornwood, NY). Top and side view images were used to get an aspect ratio (width/thickness) of chondrocyte microtissues. Each microtissue was then individually released from its gel and loaded into a laser cut polymethyl methacrylate (PMMA) frame, and the microtissues were dusted with ground allspice to track local variations in strain. Due to the high water content of our samples, we eliminated the use of soluble dyes that might diffuse or insoluble paints with toxic vehicles (24). Instead, samples were speckled on their surfaces with allspice. The frame was then loaded into the 
grippers of a custom built mechanical testing device and the edges of the frame were cut to allow for direct tissue loading. The custom built mechanical testing setup, described previously (25), consisted of a centrally positioned linear actuator (Series A, Ultramotion) with a built in encoder, which recorded motor displacement to determine the crosshead displacement. Coupled to the moving end of the linear actuator was a 50 gram tension/compression force transducer (LCFA50G, Omega Engineering Inc.) which provided force measurements during the tensile test. Force measurements were converted into nominal engineering stresses via the measured initial crosssectional area for each specimen. The apparatus was controlled by a custom LabVIEW VI program (National Instruments Corporation, Austin, TX, USA). A loading rate of $0.05 \mathrm{~mm} / \mathrm{s}$ was used for the dogbones seeded in the $600 \mu \mathrm{m}$ mold $(\mathrm{n}=5)$ and at both $0.05 \mathrm{~mm} / \mathrm{s}(\mathrm{n}=7)$ and 0.1 $\mathrm{mm} / \mathrm{s}(\mathrm{n}=4)$ for the dogbones seeded in the $400 \mu \mathrm{m}$ mold. Tissue strain was visually recorded using a Canon Eos Rebel T3i camera. To analyze local tissue strain over the course of tensile loading, marker displacement was measured at various locations along the dogbone microtissue with respect to a fixed origin ( $\mathrm{n}=6,400 \mu \mathrm{m}$ samples only). Local strain was calculated as the change in distance between successive particles divided by the initial inter-particle distance.

Stress analysis was computed using MATLAB (The MathWorks, Inc., Natick, MA, USA). True strength was calculated from the maximum force and final cross-sectional area of the tissue at the location of failure. "Average engineering strength of the rod" was calculated from the maximum force and the initial average cross-sectional area of the rod of the tissue. "Minimum strength of rod" was calculated from the maximum force and final cross-sectional area of the thinnest portions of the rod. For all samples, a bulk tangent modulus was calculated from the slope of the stress-strain curves (average engineering strength plots) for the last $10 \%$ of 
tissue strain as this coincided with the linear region of the curve. As a control, Sylgard 184 polydimethylsiloxane (PDMS) was cast at a 10:1 (base:curing agent) ratio and cut into thin strips and subjected to mechanical loading at $0.05 \mathrm{~mm} / \mathrm{s}$.

From strain mapping, force data, and initial tissue dimensions, the average engineering stress was calculated for the different regions of the dogbone tissue. To determine the modulus for each region of the tissue, a linear regression was performed for the linear portion of the stress-strain curve.

To determine if mold width (400 or $600 \mu \mathrm{m})$ and loading rate $(.05$ or $.1 \mathrm{~mm} / \mathrm{s})$ independently had an effect on tissue strength, "average engineering strength of the rod," "minimum strength of rod," and bulk tangent moduli, student t-tests (two-tail, unequal variance), were performed with a $95 \%$ confidence interval.

\section{Principle Stress Modeling}

Stress analysis for the toroid and dogbone molds has been previously described $(26,27)$. For each of the new mold designs, the stresses in microtissues were computed in the finite element framework using the package ABAQUS v6.10 (SIMULIA, Providence, RI) and was based on the micro-mold dimensions. The model contained two parts: the posts and the tissue. The posts were treated as rigid body, as they are much stiffer than the tissue, and the tissue was modeled as a neo-Hookean material. The interaction between tissue and the posts were treated as frictionless contacts. During the cellular self-assembly process, the contractile cells cooperated with each other and therefore generated tissue level tension. To mimic this process, homogeneous contraction was applied to the tissue portion of the model until it reached the final 
shape observed experimentally. The maximal principle stress was plotted for the contracted state to show the stress in tissue level and the maximal principle stress direction in certain regions was plotted to show the local stress direction. 


\section{Results}

In directed self-assembly, the obstacles in a non-adhesive micro-mold induce tension in the self-assembling 3D tissue. To determine to what extent mold design controls this tension, we designed five different micro-molds to create tissues with uniaxial tension (loop-ended dogbone tissues), circumferential tension (toroid tissues), biaxial tension (cross tissues), equibiaxial tension (trampoline tissues), and multiaxial tension (triangle tissues) (Figure 1A-E). The mold designs were modeled using ABAQUS to compute the maximal principle stress (Figure 1F-J) and the local stress directions (Figure 1K-O). Simulation results indicate that the direction of the local stress varies for each designs.

To confirm that the different designs can direct cell-mediated tension, we seeded normal human chondrocytes into the non-adhesive micro-molds. The cells aggregated, rapidly selfassembled, and generated tension (Figure 2A-E, Supplemental Video 1). Within 24 hours, the cells had self-assembled a stable microtissue, with the tension continuing to mount through 96 hours (Supplemental Videos 2 and 3).

To investigate if the different mold designs caused cells to align in the predicted directions, chondrocyte microtissues were matured for four days, fixed, and stained for nuclei and F-actin (Figure 2F-J). From confocal images, we quantified the angle of the major axis of the nuclei in the five different designs (Figure 3). For the dogbone microtissue, it was predicted the cells would align uniaxially, thus parallel with the horizontal. The analysis of nuclei showed that $72 \pm 13 \%$ of the cells were aligned between $0^{\circ}$ to $14^{\circ}$ from the horizontal with an average nuclei angle of $10 \pm 3^{\circ}$. For the cross microtissue, it was predicted the cells would align around $0^{\circ}$ and $180^{\circ}$ in the left and right arms of the microtissue and that the cells would align at $90^{\circ}$ in the top and bottom arms. Nuclei analysis indicated that cells aligned at these angles with peaks in 
the nuclei distribution at $0-30^{\circ}$ and $150-180^{\circ}$ for the horizontal arms, and between $90-120^{\circ}$ for the vertical arms $(\mathrm{p}<.05)$. The trampoline microtissue was predicted to have equibiaxial tension, with cells aligning equally along all directions. Nuclei analysis showed a random arrangement of cell alignment in the $x-y$ plane $\left(0-180^{\circ}\right)$, with no statistically significant difference in the number of cells within each angle bin. For the triangle microtissue with peg constraints at the vertices of an equilateral triangle, alignment was predicted along the arms of the tissue at $60^{\circ}$. Analysis showed that the nuclei in the $30-60^{\circ}$ angle bin and the $60-90^{\circ}$ angle bin were statistically more than the other angle bins $(\mathrm{p}<.05)$, but not significantly different from each other, with the nuclei aligned at $72 \pm 12^{\circ}$.

To determine if mold design's control over cell alignment also extended to the alignment of the extracellular matrix (ECM) proteins synthesized by the cells, we stained for collagen II. Chondrocytes were seeded into the toroid, dogbone, cross, trampoline, and triangle molds and after 4 weeks, the resulting microtissues were fixed and immunostained for collagen II. In all five of the mold designs, the chondrocytes produced collagen II (Figure 2K-O) that was aligned in the direction corresponding to the nuclei.

In self-assembled tissues, the type of ECM synthesized is determined by the cells which are present. To establish if mold design can control the alignment of other cell types that synthesize different ECM proteins, we tested normal human fibroblasts (NHFs). NHFs were seeded into dogbone and trampoline molds and matured for 4 weeks. Similar to chondrocytes, NHFs self-assembled into complex shaped tissues. However, as a more contractile cell type, the gross morphology of the NHF tissue differed slightly from that of the chondrocytes. Specifically, NHF dogbone microtissues had more cell elongation and as a result created a thinner connecting rod in the dogbone tissue, whereas the trampoline tissues had a higher density of cells in the 
central portion of the tissue (Figure 4A-B). These tissues were fixed and immunostained for elastin (Figure 4-D). Confocal imaging revealed that in a self-assembled environment, these NHFs produced elastin and that both the nuclei and elastin were aligned in the direction set through mold design. Specifically, these tissues produced uniaxially aligned elastin fibers in the dogbone mold, and unaligned elastin in the trampoline mold.

To determine if there was collagen I present in our chondrocyte microtissue, all chondrocyte microtissue were stained for collagen I (Supplemental Figure 1). Only dogbone samples stained positive for collagen I and the staining was only at the surface of the tissue and not in deeper optical slices. NHF dogbones were also stained for collagen I (Supplemental Figure 2). Collagen I staining was much more diffuse than the elastin staining for our NHF tissues which follows previous studies that showed that when NHFs are cultured long term, under tension, with TGF- $\beta$, they have diminished collagen I production and enhanced elastin production (28).

To create larger tissues, we designed a scaled-up trampoline mold. This mold had a hexagonal central trough surrounded by 3 orbitals of posts (around the hexagon's perimeter) which act as a constraint to the central portion of the tissue. This design had a contiguous tissue that measured $1.1 \mathrm{~cm}$ in its largest dimension and had a sheet of tissue $22 \mathrm{~mm}^{2}$ in area. Chondrocytes rapidly self-assembled into this large trampoline structure. Although there were some points of tissue failure located near the posts, the central portion of the tissue was stable through eight weeks. After eight weeks, the tissue was fixed and immunostained for collagen II. Similar to the smaller trampoline structure, there was no preferential alignment of the nuclei, and collagen II was present and also unaligned (Figure 5). 
To understand the complexity of the mechanical properties of the self-assembled tissues, chondrocytes were seeded into dogbone micro-molds with connecting rod widths of 400 and 600 $\mu \mathrm{m}$. These samples were matured for 8 weeks, speckled, and subjected to uniaxial tensile testing at a loading rate of $0.05 \mathrm{~mm} / \mathrm{s}$ for the dogbones seeded in the $600 \mu \mathrm{m}$ mold and at both 0.05 $\mathrm{mm} / \mathrm{s}$ and $0.1 \mathrm{~mm} / \mathrm{s}$ for the dogbones seeded in the $400 \mu \mathrm{m}$ mold (Figure 6A and Supplemental Video 2). Although the dogbone tissues as a whole underwent $55.3 \pm 6.2 \%$ strain (as measured by motor displacement) there were local variations in the mechanical behavior of the tissue. By measuring the displacement of the speckle markers over the duration of mechanical loading, we were able to calculate the local strain within different portions of the tissue. We identified three distinct regions of the tissue: the rod portion, the toroid portion, and the region connecting the rod portion to the toroid portion ("Y-junction"). Each of these regions underwent different levels of strain under tensile loading (Figure 6B). For all of the samples, the rod portion had the least amount of strain before failure, experiencing strains of $20.8 \pm 8.0 \%$, while the locations surrounding the Y-junction experienced the highest levels of strain $(71.6 \pm 8.0 \%)$. Overall, while the rod portion of the dogbone was very homogeneous, experiencing $29.1 \pm 10.7 \%$ strain through the length of the tissue, the dogbone tissue as a whole was heterogeneous in strain behavior when comparing the stiff rod region to the other two regions $(3.6 \pm 1.3$ fold difference in strain). Interestingly, the three regions of the dogbone tissue have different nuclei and collagen II alignment as the collagen goes from circumferentially aligned in the toroid portion, to a forked alignment in the Y-junction, to uniaxially aligned in the rod portion (Supplemental Figure 3).

To elucidate the stress response of the dogbone tissue to uniaxial loading, we generated stress-strain curves from the motor displacement, initial tissue dimensions, and load cell readings. The stress-strain curve of our microtissues followed the same pattern of what is seen 
with soft biological tissues (Figure 6C) with an initial toe-region, a linear region, and then a failure region. From the average initial cross-sectional area of the rod portion of the tissues, we calculated the average engineering stresses experienced by the rod portion of the tissue (Figure 6C, Table 1). Coinciding with the pattern of strain, the samples consistently failed at the Yjunction. These areas had the lowest degree of collagen alignment (Supplemental Figure 3) and so intuitively would coincide with the weakest portion of the tissue. From the final crosssectional area of the tissue at the region of failure, the true strength of the tissue was calculated for each test condition. Interestingly, the samples did not fail at the thinnest portion of the tissue (rod region), but at the thick, forked region of the tissue (Y-junction). Since the samples did not fail in the rod portion of the tissue, using the maximum force data, we calculated what would be the "minimum strength" of the thinnest part of the tissue (the rod) (Table 1). For the $400 \mu \mathrm{m}$ samples, there was no difference in minimum strength of the rod portion of the tissue with the different strain rates, but there was a difference between the 400 and $600 \mu \mathrm{m}$ samples. The 400 $\mu \mathrm{m}$ samples exhibited up to $2 \mathrm{X}$ more strength than the $600 \mu \mathrm{m}$ samples. These $400 \mu \mathrm{m}$ dogbones were only $156 \pm 58 \mu \mathrm{m}$ in width and were withstanding $39 \pm 11 \mathrm{mN}$ of tensile force without failing or even necking. By comparing the true strength of the Y-junction of the tissue to the minimum strength of the rod region of the tissue, there was at least a 3.5 and 4.5 fold difference in the strength of these regions for the $400 \mu \mathrm{m}$ and $600 \mu \mathrm{m}$ samples, respectively. To compare our tissues with those in the literature, we calculated the bulk tangent moduli from the slope of the linear portion of the stress-strain curves (average engineering strength plots). There was no difference in the moduli for the two strain rates of the $400 \mu \mathrm{m}$ samples, but the $400 \mu \mathrm{m}$ samples were stiffer than the $600 \mu \mathrm{m}$ samples. The stiffness of these dogbones were in the range of 
biological tissues and was stiffer than comparable sized strips of PDMS (measured here to be $1.23 \pm .05 \mathrm{MPa})$ 

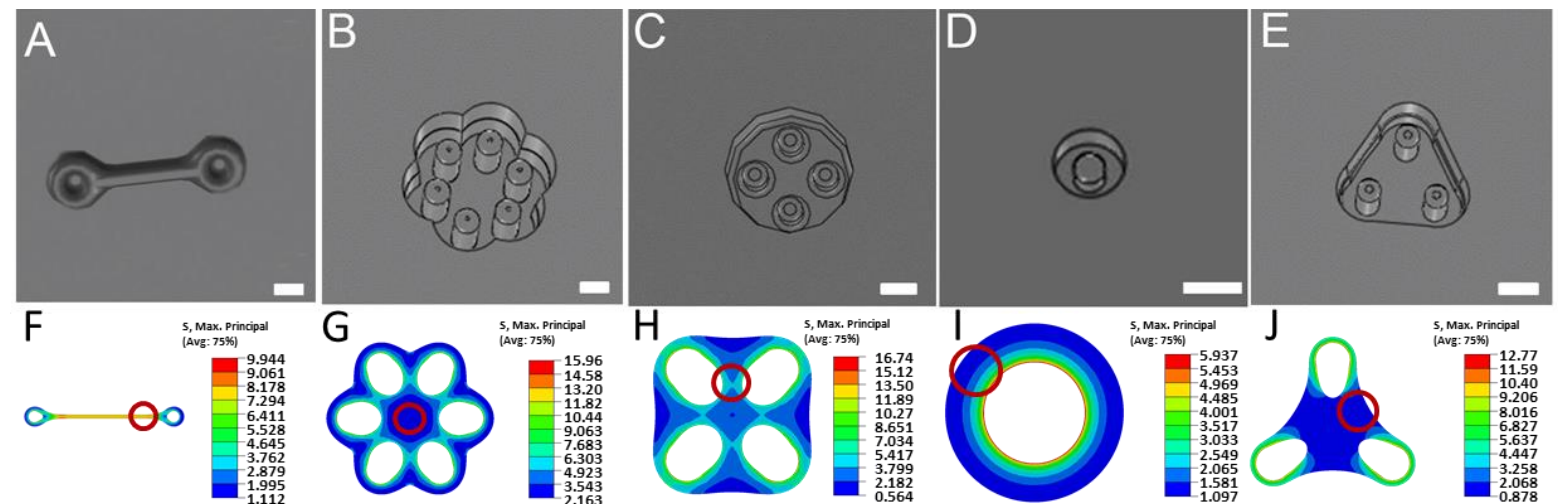

K

$\mathrm{L}$
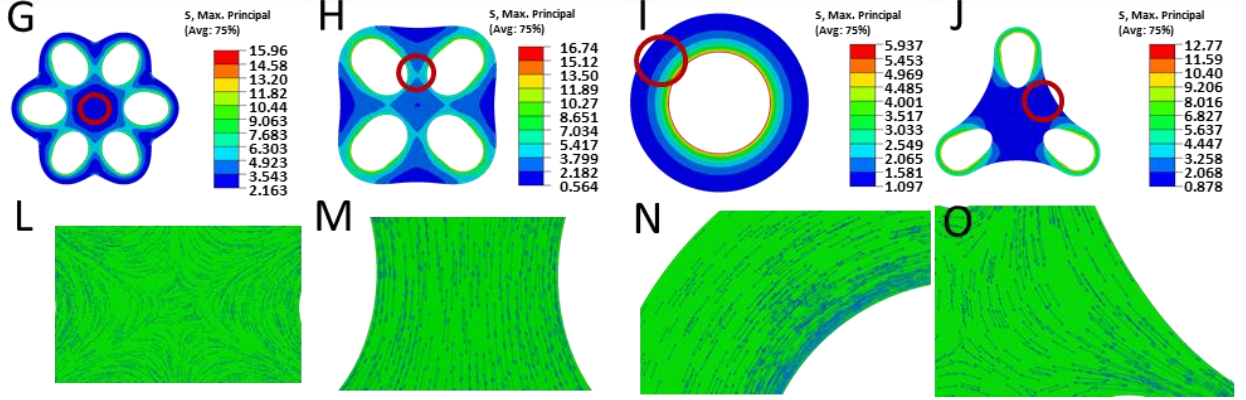

Figure 1: Micro-molds were designed (A-E) to harness cell-derived forces to create uniaxial (dogbone; A, F, K), equibiaxial; (trampoline; B, G, L), biaxial (cross; C, H, M), circumferential (toroid; D, I, N), and multiaxial tension (triangle; E, I, O) within the self-assembled tissue. Maximal principle stress (F-J) and max principle stress direction $(\mathrm{K}-\mathrm{O})$ were modeled for each of the mold designs. Scale bar is $1 \mathrm{~mm}$.
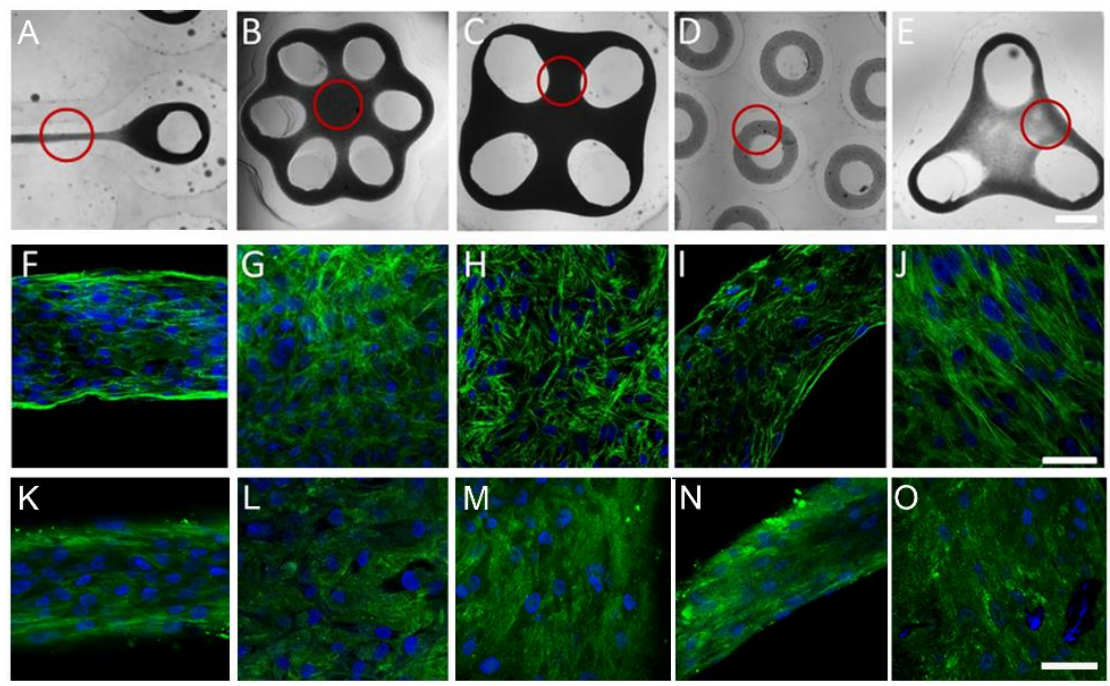

Figure 2: Human chondrocytes rapidly self-assemble in micro-molds, exert cell-derived forces, align, and produce aligned collagen II. Chondrocytes were seeded into micro-molds designed to create uniaxial (A,F,K), equibiaxial $(\mathrm{B}, \mathrm{G}, \mathrm{L})$, biaxial $(\mathrm{C}, \mathrm{H}, \mathrm{M})$, circumferential $(\mathrm{D}, \mathrm{I}, \mathrm{N})$, and multiaxial $(\mathrm{E}, \mathrm{J}, \mathrm{O})$ tension. After four days in culture, microtissues were fixed, their nuclei were labeled with DAPI (blue), their f-actin was labeled with a fluorescent Phalloidin (green), and they were imaged with confocal microscopy (F-J). For each of the mold designs, cells aligned according to the direction of stress. After four weeks of maturation, samples were labeled for collagen II (green) and DAPI (blue) (K-O). Synthesized collagen II followed the cellular alignment. Scale Bar is $300 \mu \mathrm{m}$ in panel $\mathrm{E}$, and $100 \mu \mathrm{m}$ in panels $\mathrm{J}$ and $\mathrm{O}$. 

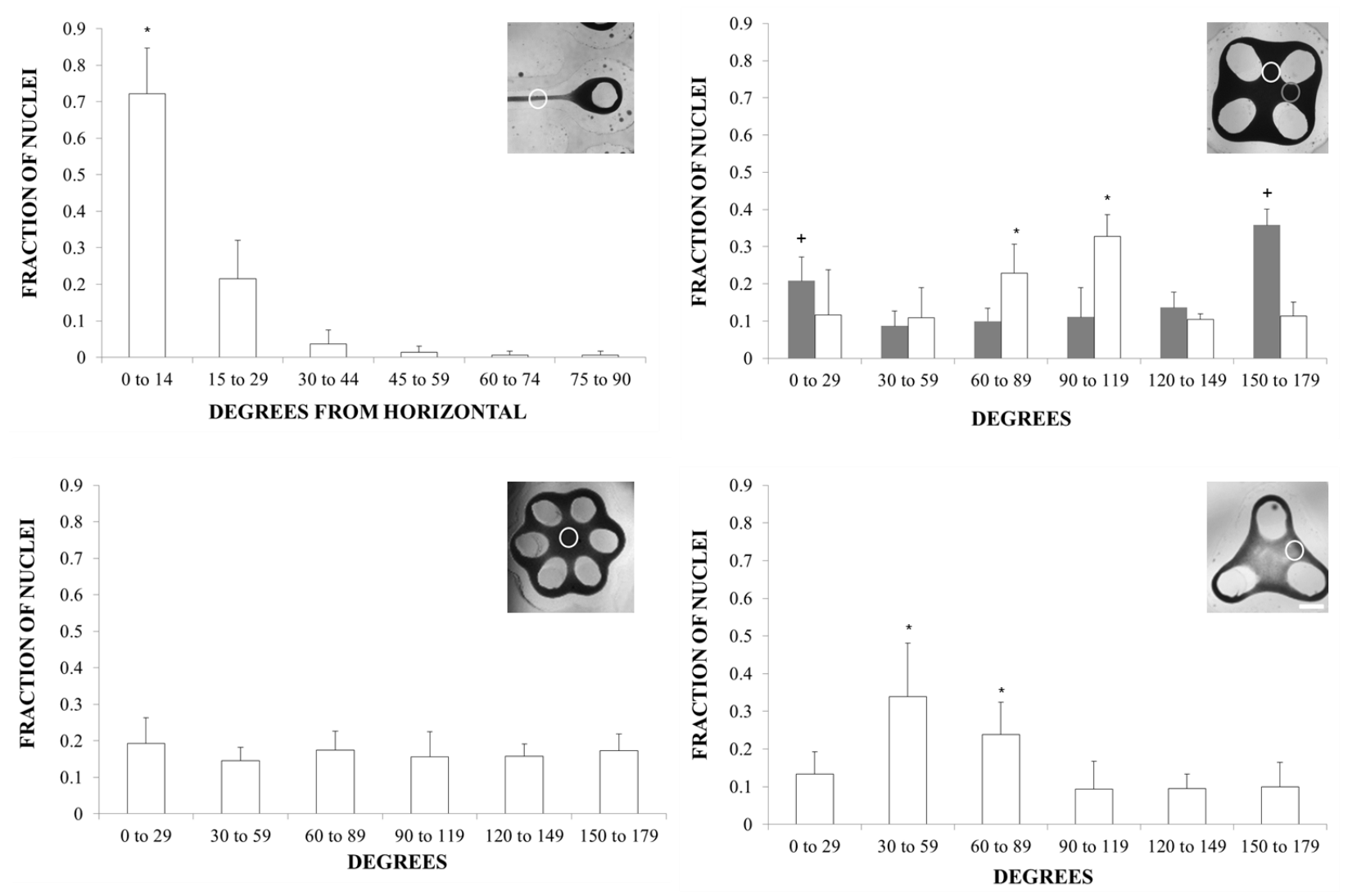

Figure 3: Micro-mold design controls nuclei alignment creating four distinct cell alignments. The angle of the major axis of the nuclei of cells within the dogbone (A), cross (B), trampoline (C), and triangle (D) microtissues were quantified and binned in $15^{\circ}$ bins for the dogbone, and $30^{\circ}$ bins for the three other shapes. Each shape had more nuclei aligned nuclei in the bins predicted for each shape ( $\mathrm{p}<.05)$. Specifically nuclei aligned between $0^{\circ}$ to $14^{\circ}$ from the horizontal in the rod portion of the dogbones; $0^{\circ}$ and $180^{\circ}$ (gray bars) for the horizontal portions of the cross (gray circle in inset) and $90^{\circ}$ (white bars) for the vertical portions (white circle in inset) of the cross; and $30-60^{\circ}$ and $60-90^{\circ}$ leg of the triangle. The fraction of nuclei in each of the bins for the trampoline was not statistically different indicating that there was equibiaxial cell alignment. 

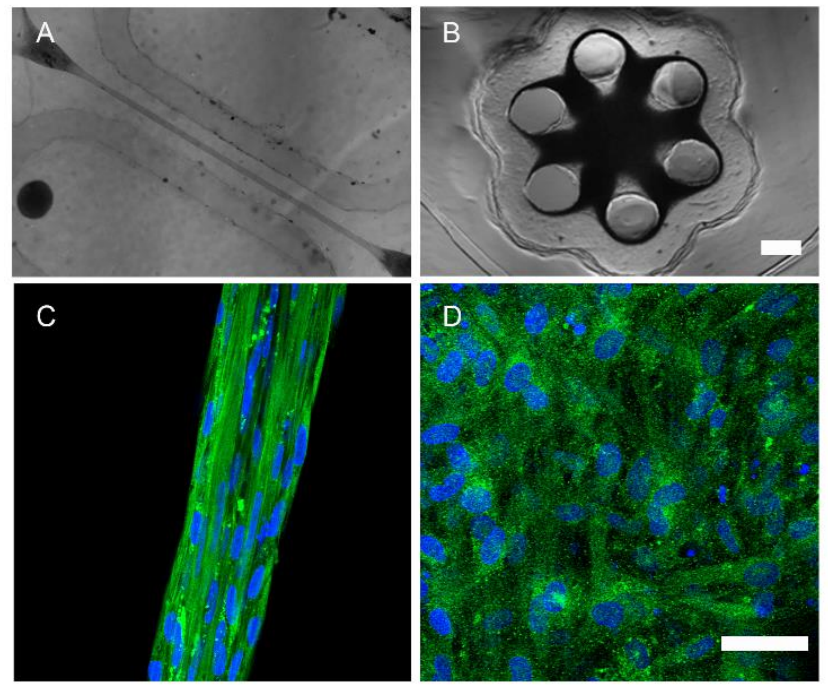

Figure 4: Self-assembled NHF microtissues produce elastin in uniaxial dogbone molds and equibiaxial trampoline molds. NHF dogbones $(A, C)$ and trampolines $(B, D)$ were matured for four weeks, fixed, the nuclei were labeled with DAPI (blue), and the tissue was stained for elastin (green). Within the dogbone and trampoline molds, NHFs produces uniaxially and equibiaxially aligned elastin, respectively. Scale bars in B and D are 500 and $50 \mu \mathrm{m}$, respectively.
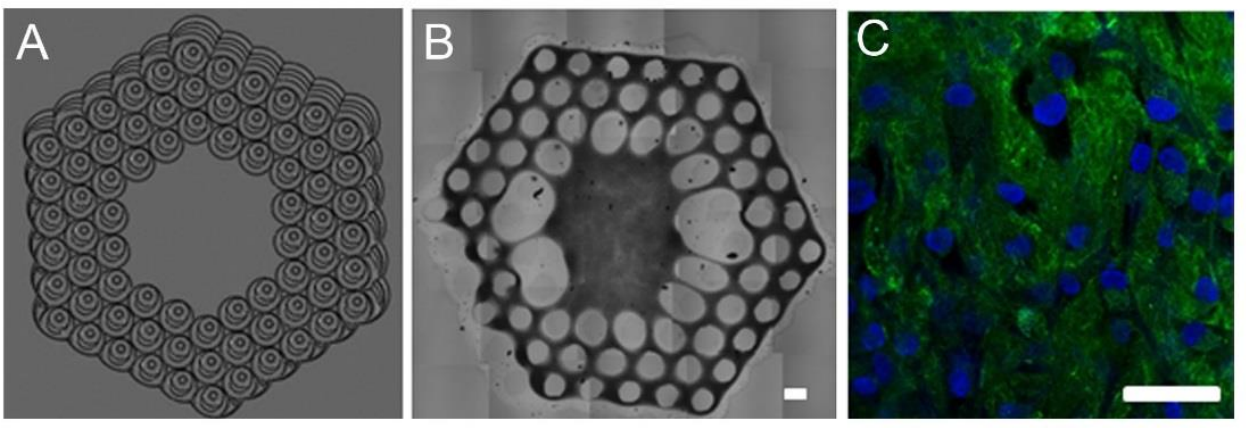

Figure 5: Chondrocytes form a scaled-up self-assembled trampoline structure while preserving equibiaxial cell and collagen II alignment. A scaled up $(1.1 \mathrm{~cm})$ mold was designed to create a large tissue with equibiaxial alignment (A). Chondrocytes self-assembled a tissue that remained stable through eight weeks of culture (B). The nuclei were labeled with DAPI (blue) and stained for collagen II (green). Confocal imaging demonstrated that collagen II fibers and cell nuclei (C) were randomly aligned through the tissue. Scale bar is $50 \mu \mathrm{m}$. 


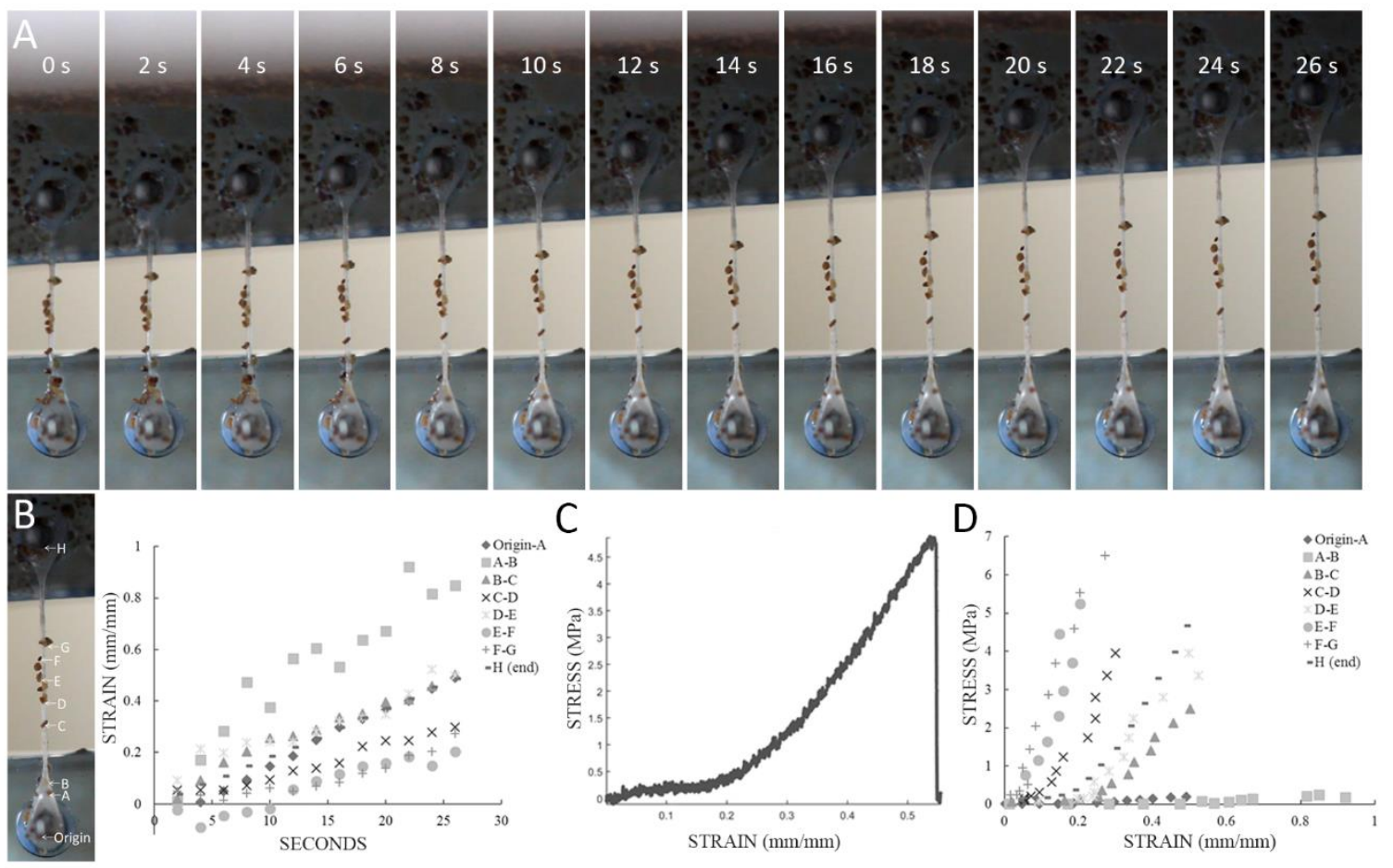

Figure 6: Self-assembled dogbone microtissues under tensile loading exhibit heterogeneous strain behavior according to the region of the tissue. Dogbone samples were speckled, loaded into a custom tensile tester, and loaded at a rate of $0.1 \mathrm{~mm} / \mathrm{s}(\mathrm{A})$. Marker displacement was measured over time for each sample and the strain between markers was computed over the duration of mechanical testing. (B) Dogbone microtissues exhibited heterogeneous strain by region. Specifically, the dogbone toroid to Y-junction region ("A-B") and Y-junction to rod region ("C-D"), exhibited much higher levels of strain than the pure rod portions ("D-E", "E-F", "F-G") of the tissue. Stress was calculated from the initial cross-sectional area of the rod portion of the tissue. Shown is a representative stress-strain curve of dogbone under tensile loading (C). From strain mapping, force data, and initial tissue dimensions, the average engineering stress was calculated for each region of the tissue. Shown is a representative stress-strain plot by region (D). 
Table 1: Self-assembled dogbone microtissues possess mechanical properties in the range of natural tissues. Chondrocytes were seeded in $600 \mu \mathrm{m}$ and $400 \mu \mathrm{m}$ molds, matured for eight weeks, and were strained at $.05 \mathrm{~mm} / \mathrm{s}$ (low $\dot{\varepsilon}$ ) and $.1 \mathrm{~mm} / \mathrm{s}$ (high $\dot{\varepsilon}$ ). True strength was calculated from the maximum force and final cross-sectional area at the location of failure (I)(B). "Average engineering strength of the rod" was calculated from the maximum force and the initial average cross-sectional area of the tissue (II)(A). "Minimum strength of rod" was calculated from the maximum force and final cross-sectional area of the thinnest portions of the rod (III) (B). Bulk moduli were calculated from the slope of the upper linear region of the stress-strain curves (average engineering strength plots). *Indicates a significant difference $(\mathrm{p}<0.05)$ with the $600 \mu \mathrm{m}$ tissues.

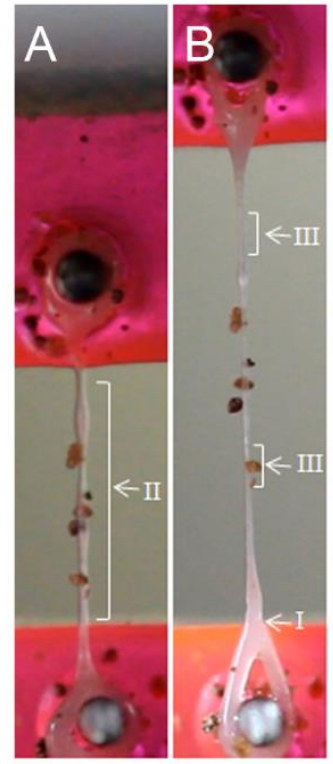

\begin{tabular}{|c|c|c|c|c|}
\hline & $\begin{array}{c}600 \mu \mathrm{m}, \text { low } \dot{\varepsilon} \\
\text { (MPa) }\end{array}$ & $\begin{array}{c}\text { 400 } \mu \mathrm{m} \text {, all } \dot{\varepsilon} \\
\text { (MPa) }\end{array}$ & $\begin{array}{c}400 \mu \mathrm{m}, \text { low } \dot{\varepsilon} \\
\text { (MPa) }\end{array}$ & $\begin{array}{l}400 \mu \mathrm{m}, \text { high } \dot{\varepsilon} \\
\text { (MPa) }\end{array}$ \\
\hline $\begin{array}{c}\text { True } \\
\text { Strength of } \\
\text { Dogbone (I) }\end{array}$ & $1.13 \pm 0.44$ & $2.85 \pm 1.94$ & $2.57 \pm 1.47$ & $3.35 \pm 2.77$ \\
\hline $\begin{array}{c}\text { Engineering } \\
\text { Strength of } \\
\text { Rod (II) }\end{array}$ & $2.65 \pm 1.07$ & $4.62 \pm 1.95^{*}$ & $4.34 \pm 2.11$ & $5.11 \pm 1.81 *$ \\
\hline $\begin{array}{l}\text { Minimum } \\
\text { Strength of } \\
\text { Rod (III) }\end{array}$ & $5.08 \pm 2.39$ & $9.17 \pm 3.41 *$ & $10.25 \pm 3.85^{*}$ & $8.45 \pm 1.45^{*}$ \\
\hline Modulus & $8.80 \pm 3.22$ & $15.85 \pm 6.25^{*}$ & $14.58 \pm 6.06$ & $18.8 \pm 6.8^{*}$ \\
\hline
\end{tabular}




\section{Discussion}

The extracellular matrix (ECM) and the alignment of cells play important roles in the structure and function of a tissue. In vivo, there is a range of ECM alignments from uniaxial aligned collagen, as is found in tendons and ligaments, to unaligned collagen, as is found in the dermis, to collagen arranged at prescribed angles, as is found in small intestine submucosa and the myocardium. These different alignments, in addition to the specific composition and blend of ECM proteins, function to give tissues specific mechanical properties (strength, extensibility, stiffness, etc.) as well as physiological functions.

Numerous methods have been developed to control the alignment of various biopolymers and biocompatible polymers with or without the aid of living cells. Polymers that have been aligned include poly (lactic-co-glycolic) acid (PLGA), fibrin, and silk and many studies have focused on collagen type I because of its abundance in the body and availability as a purified protein. Methods to align solubilized collagen monomers without the aid of cells include electrospinning (29), external magnets (1, 30), fluid flow (31), or extrusion (32). Alignment of purified collagen using these mechanical means do not yield material with sufficient mechanical strength so often these materials are chemically cross-linked (e.g., genapin, carbodiimide) which can result in problems with biocompatibility (33-35).

It has been known for many years that living cells can aid in the alignment of collagen monomers if the cells are cast into the gel. Fibroblast seeded into collagen lattices will contract and compact the collagen gel. The cells remodel the exogenous collagen of the gel as they synthesize their own endogenous collagen along with the other ECM proteins they synthesize. Obstacles that interrupt the collagen gel as well as how the collagen gel is tethered (boundary conditions), can modulate how the cells align the collagen (2, 36-38). There are, however, 
several significant differences between cells reorganizing a collagen gel and native tissues. First, the protein content of a collagen gel is relatively low (0.1-0.5\% by weight) compared to native tissues (i.e. skin, $>5 \%$ )(39). As the cells reorganize the collagen gel, they are not reconstituting collagen's true native hierarchical structure. The cells are reorganizing the collagen fibrils by simple end-to-end and lateral assembly. Lastly, the cells do synthesize endogenous ECM, but even after four weeks, at least $20 \%$ of the original collagen is still present (39). As a result of the residual collagen, its non-hierarchical assembly, and the low protein content, these remodeled collagen gels are at least an order of magnitude weaker than in vivo tissues even when subjected to additional external mechanical conditioning techniques, such as cyclic stretch (40).

In this study, we have utilized the cells' innate capacity to form ECM, their contractile nature, and mold design to create strong tissues with both cell and collagen alignment. We have engineered five different mold designs to harness cell-derived contractile forces, thereby creating five distinct tension environments. We seeded mono-dispersed cells into these molds made of non-adhesive hydrogels to create tissues with uniaxial, circumferential, biaxial, equibiaxial, or multiaxial tension. After settling into the non-adhesive micro-molds, the cells were unable to attach to the hydrogel and so formed cell-cell adhesions and exerted contractile forces that drove the self-assembly of a 3D multi-cellular microtissue. The micro-mold design sets the initial position of the cells and provides constraints which direct cell alignment and tissue tension which ultimately directs ECM alignment. Unlike traditional tissue engineering, our tissues were created without exogenous ECM proteins or scaffold and without the use of external mechanical stimulation. Even though our microtissues are initially composed of only cells, after maturation, these tissues have a dense accumulation of aligned collagen II fibers, and have a strength and moduli in the range of soft tissues. 
We hypothesize that our tissues are stronger than collagen gels with cells, and better match the mechanical properties of native tissues because the 3D self-assembled environment more closely matches the in vivo environment. In vivo, collagen is produced by cells from the "bottom-up" starting on the molecular level forming fibrils and then fibers (9). The interactions that occur between the different collagen substructures, the organization, and the cross-linking within each fibril are thought to be critical to the overall physiological and mechanical properties of the tissue (39). As well, this process of the cells synthesizing EMC renders a specific fibril/fiber packing and weave that entraps cells and gives tissues their unique properties. The fact that the cells within our self-assembled structures are using this "bottom-up" approach to form a complex interconnected collagen network means that the collagen has the hierarchal structure and natural cross-links found in vivo. This is corroborated in that shape of the stressstrain curves of our dogbone microtissues closely resemble that which is seen for soft tissues like tendons and ligaments (39). As well, in vivo as cells synthesize collagen, they interweave other types of nonfibrillar collagen and other ECM molecules at specific intervals which modulates ECM function. For example, proteoglycans have been shown to control the size of collagen fibers formed in vivo, which also has a role in the strength, stress dissipation, and elasticity of a tissue (41). Most tissue engineered constructs are fabricated from purified collagen, while some researchers have incorporated glycosaminoglycans (42), and others have co-spun collagen and elastin (3). However, these supplemental proteins and molecules cannot be incorporated with the same level of organization and specificity as is done naturally by the cells synthesizing the ECM and as a result, cannot reconstitute the native ECM. Here, our self-assembled chondrocyte microtissues were extremely hydrophilic, suggesting the presence of cell synthesized proteoglycans, which may have also played a role in the superior tissue mechanics. Other groups 
have also shown that self-assembling chondrocytes produce other ECM proteins in addition to collagen II (17).

Further recapitulating the in vivo environment, our tissues have maximized cell-cell interactions. Cell-cell adhesion and communication are known to be pivotal in a number of cell functions. For example, mechanical stimuli is transmitted through adherens junctions, and this force transmission has been shown to initiate a host of cell signaling pathways including the formation of stress fibers and the upregulation of ECM synthesis (43). We hypothesize that since the forces acting on the cells in our tissues are endogenous cell-derived forces (as compared to applying external stretch/compression), these forces are at a more physiologically relevant level, and so would result in an environment that restores cell phenotype and promotes ECM production. These differences in mechanical stimuli, cell-cell communication, and collagen synthesis could account for the significant increase in strength that is seen in our self-assembled structures as compared to those structures made from collagen gels with cells.

Not only does this method create tissues with relevant tensile strengths and moduli, but it gives us the ability to truly engineer and control the material's biological composition as well as mechanical properties. Using this technology, we have the ability to control and tailor which ECM proteins are formed in vitro to fulfill specific applications. Although this study focused on chondrocytes and fibroblasts, our goal was not to engineer cartilage, but rather to show that by choosing a specific cell type, we can dictate the proteins that are formed, in this case collagen II and elastin. By this, we are demonstrating that cell type selection is what determines the type of ECM that is created in self-assembled tissues. To further tailor biological composition, performance, and mechanical properties, we can foresee co-seeding different cell types to create tissues with blends of ECM. By mixing fibroblasts with chondrocytes at different ratios we could 
tune mechanical properties (i.e. change elastic properties, increase tensile strength) as collagen (produced by chondrocytes) gives strength to a tissue, while the elastin (produced by the fibroblasts) gives a tissue more elasticity (28). We have also previously demonstrated that we can control how the cells in heterotypic self-assembled tissues organize, manipulating the cells innate propensity to self-sort, and so we could also create layered or blended tissues $(44,45)$.

As it is well known that fiber alignment dictates mechanical performance, this technology is well suited for creating tissues with specific geometries and thus tailored mechanical properties. In this study, by simply altering the width of the connecting rod of the dogbone micro-mold by $33 \%$ we created approximately a $200 \%$ increase in both tissue strength and modulus, demonstrating the degree to which mold geometry can tailor mechanical properties. As well, within our dogbone microtissues, we have shown the importance of cell and ECM alignment in the modulation of the mechanical properties of self-assembled tissues, as the less aligned portions of the tissue exhibited only about $20 \%$ of the strength and stiffness of the highly aligned rod portion of the tissue. The difference in mechanical properties within a single microtissue is of biological relevance as, for example, single cartilage samples have exhibited a twofold difference in shear modulus over a length of only about $100 \mu \mathrm{m}$ (46). Similarly, the authors of this study concluded that this sharp difference in mechanics is due to collagen alignment. Here, we used FEA only to model the five different tension environments of our molds. However, FEA could be further utilized to predict tension environments and then inform the design of a number of new molds, with different geometries, for specific tissue engineering applications. 


\section{Conclusion}

A persistent challenge to the utility of tissue engineering is the lack of strong, natural tissues, with relevant blends and alignments of both ECM and cells. Polymers can be highly aligned and are strong, but lack the important biological signals and properties of native ECM. Biopolymers, such as fibrin and collagen type I, offer a more natural solution, but without chemical cross-linking that can compromise biocompatibility, these biomaterials lack relevant mechanical properties. Directing the assembly of monodispersed cells into complex shaped tissues presents a solution to the shortcomings of synthetic polymers and purified biopolymers, while offering strong, biologically tailorable, mechanically tunable tissues that are simply made. With more self-assembled microtissues already being tested in the clinic (47), and new methods being developed to scale up the size of self-assembled tissues (48), we present here even more control and diversity to this already promising method of tissue engineering.

\section{Acknowledgments}

This work was funded in part by DoD Grant W81XWH-10-1-0643, NSF Grant 1129172, and NSF grant CBET-1428092. J. Morgan has an equity interest in Microtissues, Inc. This relationship has been reviewed and managed by Brown University in accordance with its conflict of interest policies.

\section{References}

1. Tranquillo RT, Girton TS, Bromberek BA, Triebes TG, Mooradian DL. Magnetically orientated tissue-equivalent tubes: application to a circumferentially orientated media-equivalent. Biomaterials. 1996;17(3):349-57.

2. Neidert MR, Tranquillo RT. Tissue-engineered valves with commissural alignment. Tissue Eng. 2006;12(4):891-903. 
3. Buttafoco L, Kolkman NG, Engbers-Buijtenhuijs P, Poot AA, Dijkstra PJ, Vermes I, et al. Electrospinning of collagen and elastin for tissue engineering applications. Biomaterials. 2006;27(5):72434.

4. Teh TK, Toh SL, Goh JC. Aligned hybrid silk scaffold for enhanced differentiation of mesenchymal stem cells into ligament fibroblasts. Tissue engineering Part C, Methods. 2011;17(6):687-703.

5. Morin KT, Smith AO, Davis GE, Tranquillo RT. Aligned human microvessels formed in 3D fibrin gel by constraint of gel contraction. Microvascular research. 2013;90:12-22.

6. Bell E, Ivarsson B, Merrill C. Production of a tissue-like structure by contraction of collagen lattices by human fibroblasts of different proliferative potential in vitro. Proc Natl Acad Sci U S A. 1979;76(3):1274-8.

7. Glowacki J, Mizuno S. Collagen scaffolds for tissue engineering. Biopolymers. 2008;89(5):338-44.

8. Parenteau-Bareil R, Gauvin R, Berthod F. Collagen-Based Biomaterials for Tissue Engineering Applications. Materials. 2010;3(3):1863.

9. Brown RA. Direct collagen-material engineering for tissue fabrication. Tissue Eng Part A. 2013;19(13-14):1495-8.

10. Kuo CK, Marturano JE, Tuan RS. Novel strategies in tendon and ligament tissue engineering: Advanced biomaterials and regeneration motifs. Sports medicine, arthroscopy, rehabilitation, therapy \& technology : SMARTT. 2010;2:20.

11. Crapo PM, Gilbert TW, Badylak SF. An overview of tissue and whole organ decellularization processes. Biomaterials. 2011;32(12):3233-43.

12. Badylak SF, Taylor D, Uygun K. Whole-organ tissue engineering: decellularization and recellularization of three-dimensional matrix scaffolds. Annu Rev Biomed Eng. 2011;13:27-53.

13. Kunz-Schughart LA, Schroeder JA, Wondrak M, van Rey F, Lehle K, Hofstaedter F, et al. Potential of fibroblasts to regulate the formation of three-dimensional vessel-like structures from endothelial cells in vitro. Am J Physiol Cell Physiol. 2006;290(5):C1385-98.

14. Rouwkema J, de Boer J, Van Blitterswijk CA. Endothelial cells assemble into a 3-dimensional prevascular network in a bone tissue engineering construct. Tissue Eng. 2006;12(9):2685-93.

15. Kelm JM, Ittner LM, Born W, Djonov V, Fussenegger M. Self-assembly of sensory neurons into ganglia-like microtissues. J Biotechnol. 2006;121(1):86-101.

16. Desroches BR, Zhang P, Choi BR, King ME, Maldonado AE, Li W, et al. Functional scaffold-free 3D cardiac microtissues: a novel model for the investigation of heart cells. Am J Physiol Heart Circ Physiol. 2012;302(10):H2031-42.

17. Stoddart MJ, Ettinger L, Hauselmann HJ. Enhanced matrix synthesis in de novo, scaffold free cartilage-like tissue subjected to compression and shear. Biotechnol Bioeng. 2006;95(6):1043-51.

18. Napolitano AP, Chai P, Dean DM, Morgan JR. Dynamics of the self-assembly of complex cellular aggregates on micromolded nonadhesive hydrogels. Tissue Eng. 2007;13(8):2087-94.

19. Dean DM, Napolitano AP, Youssef J, Morgan JR. Rods, tori, and honeycombs: the directed selfassembly of microtissues with prescribed microscale geometries. Faseb J. 2007;21(14):4005-12.

20. Tejavibulya N, Youssef J, Bao B, Ferruccio TM, Morgan JR. Directed self-assembly of large scaffold-free multi-cellular honeycomb structures. Biofabrication. 2011;3(3):034110.

21. Youssef J, Nurse AK, Freund LB, Morgan JR. Quantification of the forces driving self-assembly of three-dimensional microtissues. Proc Natl Acad Sci U S A. 2011;108(17):6993-8.

22. Svoronos AA, Tejavibulya N, Schell JY, Shenoy VB, Morgan JR. Micro-mold design controls the 3D morphological evolution of self-assembling multicellular microtissues. Tissue Eng Part A. 2014;20(78):1134-44.

23. Baker BM, Chen CS. Deconstructing the third dimension: how 3D culture microenvironments alter cellular cues. Journal of cell science. 2012;125(Pt 13):3015-24. 
24. Kwon HJ, Rogalsky AD, Kovalchick C, Ravichandran G. Application of Digital Image Correlation Method to Biogel. Polym Eng Sci. 2010;50(8):1585-93.

25. Toyjanova J, Flores-Cortez E, Reichner JS, Franck C. Matrix confinement plays a pivotal role in regulating neutrophil-generated tractions, speed, and integrin utilization. J Biol Chem. 2015;290(6):3752-63.

26. Youssef J, Chen P, Shenoy VB, Morgan JR. Mechanotransduction is enhanced by the synergistic action of heterotypic cell interactions and TGF-beta1. Faseb J. 2012;26(6):2522-30.

27. Wang H, Svoronos AA, Boudou T, Sakar MS, Schell JY, Morgan JR, et al. Necking and failure of constrained 3D microtissues induced by cellular tension. Proc Natl Acad Sci U S A. 2013;110(52):20923-8.

28. Syedain ZH, Tranquillo RT. TGF-beta1 diminishes collagen production during long-term cyclic stretching of engineered connective tissue: implication of decreased ERK signaling. J Biomech. 2011;44(5):848-55.

29. Zhong S, Teo WE, Zhu X, Beuerman RW, Ramakrishna S, Yung LY. An aligned nanofibrous collagen scaffold by electrospinning and its effects on in vitro fibroblast culture. Journal of biomedical materials research Part A. 2006;79(3):456-63.

30. Torbet J, Ronziere MC. Magnetic alignment of collagen during self-assembly. Biochem J. 1984;219(3):1057-9.

31. Lee $\mathrm{P}$, Lin R, Moon J, Lee LP. Microfluidic alignment of collagen fibers for in vitro cell culture. Biomedical microdevices. 2006;8(1):35-41.

32. Enea D, Henson F, Kew S, Wardale J, Getgood A, Brooks R, et al. Extruded collagen fibres for tissue engineering applications: effect of crosslinking method on mechanical and biological properties. Journal of materials science Materials in medicine. 2011;22(6):1569-78.

33. Barnes CP, Pemble CW, Brand DD, Simpson DG, Bowlin GL. Cross-linking electrospun type II collagen tissue engineering scaffolds with carbodiimide in ethanol. Tissue Eng. 2007;13(7):1593-605.

34. Girton TS, Oegema TR, GrassI ED, Isenberg BC, Tranquillo RT. Mechanisms of stiffening and strengthening in media-equivalents fabricated using glycation. J Biomech Eng. 2000;122(3):216-23.

35. Lee CR, Grodzinsky AJ, Spector M. The effects of cross-linking of collagen-glycosaminoglycan scaffolds on compressive stiffness, chondrocyte-mediated contraction, proliferation and biosynthesis. Biomaterials. 2001;22(23):3145-54.

36. Sun W, Sacks MS, Scott MJ. Effects of boundry conditions on the estimation of the planar biaxial mechanical properties of soft tissues. J Biomech Eng-T Asme. 2005;127(4):709-15.

37. Costa KD, Lee EJ, Holmes JW. Creating alignment and anisotropy in engineered heart tissue: role of boundary conditions in a model three-dimensional culture system. Tissue Eng. 2003;9(4):567-77.

38. West AR, Zaman N, Cole DJ, Walker MJ, Legant WR, Boudou T, et al. Development and characterization of a 3D multicell microtissue culture model of airway smooth muscle. American journal of physiology Lung cellular and molecular physiology. 2013;304(1):L4-16.

39. Fratzl P. Collagen : structure and mechanics. xviii, 506 pages $p$.

40. Isenberg BC, Tranquillo RT. Long-term cyclic distention enhances the mechanical properties of collagen-based media-equivalents. Annals of biomedical engineering. 2003;31(8):937-49.

41. Redaelli A, Vesentini S, Soncini M, Vena P, Mantero S, Montevecchi FM. Possible role of decorin glycosaminoglycans in fibril to fibril force transfer in relative mature tendons--a computational study from molecular to microstructural level. J Biomech. 2003;36(10):1555-69.

42. Yannas IV, Burke JF, Gordon PL, Huang C, Rubenstein RH. Design of an artificial skin. II. Control of chemical composition. Journal of biomedical materials research. 1980;14(2):107-32.

43. Chen CS, Tan J, Tien J. Mechanotransduction at cell-matrix and cell-cell contacts. Annu Rev Biomed Eng. 2004;6:275-302.

44. Dean DM, Morgan JR. Cytoskeletal-mediated tension modulates the directed self-assembly of microtissues. Tissue Eng Part A. 2008;14(12):1989-97. 
45. Rago AP, Dean DM, Morgan JR. Controlling cell position in complex heterotypic 3D microtissues by tissue fusion. Biotechnol Bioeng. 2009;102(4):1231-41.

46. Buckley MR, Gleghorn JP, Bonassar LJ, Cohen I. Mapping the depth dependence of shear properties in articular cartilage. J Biomech. 2008;41(11):2430-7.

47. Athanasiou KA, Eswaramoorthy R, Hadidi P, Hu JC. Self-organization and the self-assembling process in tissue engineering. Annu Rev Biomed Eng. 2013;15:115-36.

48. Blakely AM, Manning KL, Tripathi A, Morgan JR. Bio-Pick, Place, and Perfuse: A New Instrument for Three-Dimensional Tissue Engineering. Tissue engineering Part C, Methods. 2015;21(7):737-46. 